\title{
Design of Functional Ferritin-like Proteins with Hydrophobic Cavities
}

\author{
Joe Swift, William A. Wehbi, Brenna D. Kelly, Xiaoran Fu Stowell, \\ Jeffery G. Saven and Ivan J. Dmochowski
}

\section{Supplementary Information: Denaturation Equilibria}

These are a few notes concerning the equations used to fit the equilibrium studies of the denaturation of Dps and its sequence variants.

Generically, this is a case of a protein that folds and oligomerizes to form an $n$-mer. (For Dps, $n=12$.) We'll treat the oligomerization using the simplest model where intermediate oligomerization states with $m$ protomers $(m<n)$ are essentially unpopulated. Only folded protein forms the oligomer. The equilbria are then

$$
\begin{array}{r}
U \rightarrow F ; K_{f}=\frac{F}{U} \\
n F \rightarrow F_{n} ; K_{n}=\frac{F_{n}}{F^{n}}
\end{array}
$$

where $U=[U] / M$ is the dimensionless concentration of unfolded state, $F=[F] / M$ is that for the folded state, and $F_{n}=\left[F_{n}\right] / M$ is that of the oligomer (M is units of mol/liter). Since $F=K_{f} U$ and $F_{n}=K_{n} F^{n}=K_{n} K_{f}^{n} U^{n}$, we have that

$$
\frac{F_{n}}{U^{n}}=K_{n} K_{f}^{n}
$$

The free energy for the equlibrium can then be written as

$$
\Delta G_{n f}^{*}=-R T \ln \frac{F_{n}}{U^{n}}=-R T \ln \left(K_{n} K_{f}^{n}\right)=\Delta G_{n}^{*}+n \Delta G_{f}^{*}
$$

Consider the total concentration of folded state, $F_{t}$, which is proportional to the signal associated with the folded state (via CD or Trp fluorescence)

$$
F_{t}=n F_{n}+F=\left(n K_{n} K_{f}^{n-1} U^{n-1}+1\right) K_{f} U=\left(n \frac{F_{n}}{F}+1\right) K_{f} U
$$

for two limiting cases. For $\frac{F_{n}}{F} \ll 1$, essentially none of the folded state is present as the oligomer and

$$
\frac{F_{t}}{U}=K_{f}
$$


This is just the case for a simple monomeric protein folding equilbrium.

For $\frac{F_{n}}{F} \gg 1$, essentially all the folded state is present as the oligomer and

$$
\frac{F_{t}}{U}=n K_{n} K_{f}^{n} U^{n-1}
$$

This second case is probably much more appropriate for Dps, where all or none folding is observed, and we'll assume in what follows that $\frac{F_{n}}{F} \gg 1$. Note that

$$
\frac{F_{t} / n}{U^{n}}=K_{n} K_{f}^{n}
$$

Now write this in terms of percentages (or fractions) of folded or unfolded. Let the total concentration of protein be $P_{t}=n F_{n}+F+U$, then the fraction folded and unfolded are

$$
\begin{array}{r}
f_{F_{t}}=\frac{F_{t}}{P_{t}}=\frac{n F_{n}+F}{P_{t}} \approx \frac{n F_{n}}{P_{t}} \\
f_{U}=\frac{U}{P_{t}}
\end{array}
$$

Thus $U=f_{U} P_{t}$ and $F_{t}=f_{F_{t}} P_{t}$, and

$$
\frac{F_{t} / n}{U^{n}}=\frac{f_{F_{t}} / n}{f_{U}} P_{t}^{-(n-1)}
$$

Thus we have

$$
\begin{aligned}
\Delta G_{n f}^{*} & =-R T \ln \left(\frac{F_{t} / n}{U^{n}}\right) \\
& =-R T \ln \left(\frac{f_{F_{t} / n}}{f_{U}^{n}}\right)+(n-1) R T \ln P_{t}
\end{aligned}
$$

Thus we can plot Eq. 13 vs. denaturant concentration and try to extrapolate to zero denaturant to estimate $\Delta G_{n f}^{*}$. Note that the last term involving $P_{t}$ is independent of denaturant. It may be most appropriate to report values of $\Delta G_{n f}^{*} / n$. 


\section{Supplementary Information: UV-Vis Spectra of Iron Loaded Protein}
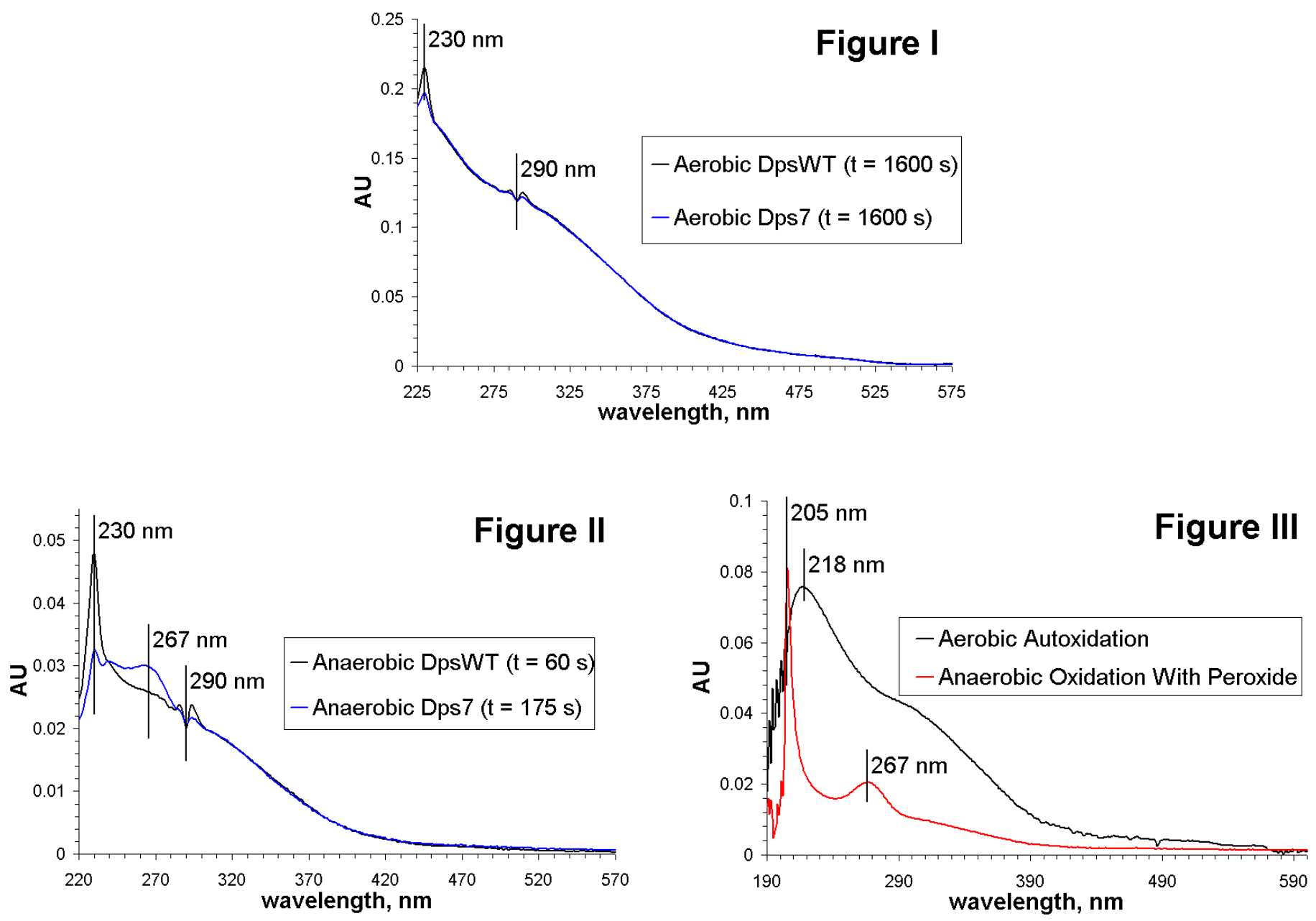

Figure I shows complete spectra at a point $1600 \mathrm{~s}$ into aerobic iron loading runs (see figure $12 \mathrm{~A}$ and the accompanying description in the experimental section of the main paper) for DpsWT and Dps7 protein. The spectra overlay almost exactly and both spectra share a point of inflection at $290 \mathrm{~nm}$. The only difference appears to be a slight reduction in the size of the maxima at $230 \mathrm{~nm}$ in the case of Dps7. Figure II shows complete spectra at points $60 \mathrm{~s}$ and $175 \mathrm{~s}$ into anaerobic iron loading runs for DpsWT and Dps7, respectively (see figure 12B in the main paper). Different time points have been chosen as iron mineralization in Dps7 proceeds at a slower rate. The spectra mostly overlay and share the same features; a point of inflection at $290 \mathrm{~nm}$ and a maximum at $230 \mathrm{~nm}$ that is suppressed in the case of Dps7. We have assigned the formation of a shoulder at $267 \mathrm{~nm}$ to a slower forming iron-peroxide species, which is observed in the case of peroxide-mediated anaerobic oxidation in the absence of protein (see Figure III). A similar shoulder was observed in the spectra of anaerobic DpsWT iron loading runs at later time points. Figure III shows complete spectra of the oxidation products of iron in the absence of protein. The aerobic autoxidation spectrum has a similar shape to the spectra of minerals produced by oxidation in the presence of protein, but lacks the maxima at $230 \mathrm{~nm}$ and the point of inflection at $290 \mathrm{~nm}$. The anaerobic oxidation of iron in the presence of peroxide shows a peak at $267 \mathrm{~nm}$. 